\title{
BMJ Open Development and validation of a risk- adjustment model for mortality and hospital length of stay for trauma patients: a prospective registry-based study in Australia
}

\author{
Arul Earnest (D) , ${ }^{1}$ Cameron Palmer, ${ }^{2}$ Gerard O'Reilly, ${ }^{1,3,4}$ Maxine Burrell, ${ }^{5}$ \\ Emily McKie, ${ }^{1}$ Sudhakar Rao, ${ }^{5}$ Kate Curtis (D) , ${ }^{6,7}$ Peter Cameron ${ }^{1,3}$
}

To cite: Earnest A, Palmer C, 0'Reilly G, et al. Development and validation of a riskadjustment model for mortality and hospital length of stay for trauma patients: a prospective registry-based study in Australia. BMJ Open 2021;11:e050795. doi:10.1136/ bmjopen-2021-050795

- Prepublication history and additional supplemental material for this paper are available online. To view these files, please visit the journal online. (http://dx.doi.org/10.1136/ bmjopen-2021-050795)

Received 04 March 2021 Accepted 05 August 2021

Check for updates

(C) Author(s) (or their employer(s)) 2021. Re-use permitted under CC BY-NC. No commercial re-use. See rights and permissions. Published by BMJ.

For numbered affiliations see end of article.

Correspondence to Professor Arul Earnest; arul.earnest@monash.edu

\section{ABSTRACT}

Objectives Adequate risk adjustment for factors beyond the control of the healthcare system contributes to the process of transparent and equitable benchmarking of trauma outcomes. Current risk adjustment models are not optimal in terms of the number and nature of predictor variables included in the model and the treatment of missing data. We propose a statistically robust and parsimonious risk adjustment model for the purpose of benchmarking.

Setting This study analysed data from the multicentre Australia New Zealand Trauma Registry from 1 July 2016 to 30 June 2018 consisting of 31 trauma centres.

Outcome measures The primary endpoints were inpatient mortality and length of hospital stay. Firth logistic regression and robust linear regression models were used to study the endpoints, respectively. Restricted cubic splines were used to model non-linear relationships with age. Model validation was performed on a subset of the dataset.

Results Of the 9509 patients in the model development cohort, $72 \%$ were male and approximately half $(51 \%)$ aged over 50 years. For mortality, cubic splines in age, injury cause, arrival Glasgow Coma Scale motor score, highest and second-highest Abbreviated Injury Scale scores and shock index were significant predictors. The model performed well in the validation sample with an area under the curve of 0.93 . For length of stay, the identified predictor variables were similar. Compared with low falls, motor vehicle occupants stayed on average 2.6 days longer (95\% Cl: 2.0 to 3.1), $p<0.001$. Sensitivity analyses did not demonstrate any marked differences in the performance of the models.

Conclusion Our risk adjustment model of six variables is efficient and can be reliably collected from registries to enhance the process of benchmarking.

\section{INTRODUCTION}

Effective, prompt, comprehensive medical care can reduce trauma-related mortality, improve outcomes and decrease the cost ('burden') of trauma to society. ${ }^{1-3}$ The

\section{Strengths and limitations of this study}

- This is a multicentre study which ensures generalisability of results across geography.

- The large sample size allows for an adequately powered study.

- Use of appropriate statistical techniques including cubic splines helps address methodological gaps.

- Sensitivity analyses was used to assess the robustness of results around key assumptions.

- Lack of simulation studies to formally compare models.

establishment of systems for trauma management within geographic regions has been demonstrated to reduce mortality ${ }^{4-6}$ within those jurisdictions, as well as comparisons made between jurisdictions without such systems in place. ${ }^{7}$ Over time, this has been achieved through assessments of quality of care. Benchmarking overall system performance, rather than individual patient mortality prediction, has been the primary aim of risk-adjustment tools. ${ }^{89}$

Early attempts to predict individual mortality risk following injury, most notably the Injury Severity Score (ISS), ${ }^{10}$ were based wholly on injury severities assigned using the Abbreviated Injury Scale (AIS) ${ }^{11} 12$ However, larger studies in the 1980s demonstrated that anatomical injury was not the only independent predictor of mortality ${ }^{13}{ }^{14}$; scores derived using the Trauma and Injury Severity Score (TRISS) methodology also employed patient age, the mechanism of injury and physiologic parameters (respiratory rate, systolic blood pressure (SBP) and Glasgow Coma Scale (GCS)) to prognosticate and do not employ optimal statistical methods. More recent studies using national-level data 
from different countries have suggested that outcome prediction may be improved by utilising these physiologic parameters in different ways ${ }^{15}$; by reassessing how overall or region-specific injury severity is described ${ }^{816}$; or by introducing new variables such as comorbid status, initial laboratory values or the use of cardiopulmonary resuscitation (CPR) prehospital. ${ }^{817}$

However, the best variables for risk adjustment or the manner in which complex data such as AIS severities should best be used remain unclear, particularly as comparisons of the performance of existing tools have returned equivocal results, and the performance of these models varies in different populations. ${ }^{18}$ This may be due in part to factors such as the case mix of each population, or differences in registry inclusion criteria, ${ }^{18}$ but the quality of the data used specifically, the level of completeness of datasets used to develop models must also be considered. The German RISC-II model attempted to account for missing data in the dataset used, ${ }^{89}$ but using a two-stage process, it is unclear how uncertainties in the estimates were incorporated in the model. Consequently, such a model may not be generalisable outside of the population in which it was developed.

If different risk-adjustment models can provide varying results, it is clearly important to develop a robust, valid, transparent and reproducible process to decide which variables to include; how to treat each variable; and how best to combine and analyse data using appropriate methods. In doing so, the process should avoid overfitting the model (ie, including too many variables), inadvertently adjusting for variables which may be influenced by the wider healthcare system (such as CPR), or including variables which are poorly measured or prone to measurement error or other types of bias.

The present study had two aims. The first was to identify significant and independent risk factors associated with inpatient mortality and hospital length of stay (LOS) among patients with trauma in Australia and New Zealand. A second aim was to develop an Australasian-specific riskadjustment model to enable benchmarking, which can be used to identify variations in care between jurisdictions or hospitals in spite of the existing data limitations. This is different from patient risk stratification or prognostication. The purpose is not to provide healthcare practitioners with real-time information, but to allow for equitable benchmarking on the trauma centres.

\section{METHODS \\ Data}

The Australia New Zealand Trauma Registry (ATR) is the collaboration of Australia and New Zealand's designated trauma centres. By combining trauma data, they are able to share expertise in trauma care and work together to both improve trauma systems and patient care through improved outcomes and quality of life in the most severely injured.
Contributing sites, of which there are 24 Australian and 7 New Zealand for the timeframe utilised in this study, provide in-hospital data on the most severely injured, categorised as an ISS $>12$ or death following injury. ${ }^{19}$ Data are submitted directly from each site or via a statebased registry. Data are submitted according to the Bi-national minimum trauma dataset. Tasmanian data are not included as they were not submitted during this timeframe. Data collection was for patients with a recorded trauma date between 1 July 2016 and 30 June 2018.

\section{Inclusion/ exclusion criteria}

Patients who were aged less than 16 at the time of trauma or sustained injuries with an ISS $<13^{20}$ were excluded from the study as they were excluded from many of the state-based registries. Only blunt injuries were included in our study, while injuries involving penetrating wounds, burns and other traumas were excluded as these are postulated to be different in aetiology and management. We also excluded patients who were transferred from other hospitals in the main analysis, but kept them for the subsequent sensitivity analysis.

\section{Data quality}

Data submitted to the ATR underwent various validity checks such as date and time formats and chronology (eg, injury date is before date of discharge from hospital, etc), and correct classification as per the International Classification of Diseases ICD-10-AM and AIS08 ${ }^{21}$ prior to data processing. Contributing sites were notified to correct any errors that were identified. ${ }^{19}$

The primary outcome measure in our study was inpatient mortality (measured as discharge destination in the registry). The coprimary outcome was LOS, measured as days between arrival at definitive care hospital to the date of discharge from definitive care, as reported by the statebased registries or individual sites.

For each endpoint, we studied the relationship of the following covariates: sex, age (in years) at date of injury, ISS, highest and second-highest AIS, ${ }^{21}$ GCS at arrival at definitive care, motor component of GCS at arrival at definitive care, cause of injury collected as an ICD-10 code and grouped into major categories similar to the Victorian Emergency Minimum Dataset, ${ }^{22}$ SBP and heart rate (HR) and shock index (SI). All variables were measured on arrival at definitive care. These variables were selected as potential risk factors as they have been established risk factors found in other studies. ${ }^{1021}$

\section{Patient and public involvement}

There was no patient or public involvement in this research as the deidentified data were used in the analysis. Patients and the public were not involved in the design, or conduct, or reporting or dissemination plans of this research.

\section{Statistical model}

Model building was based on $80 \%$ of randomly selected patient data, and model validation on the remaining $20 \%$ 
of the sample. We analysed all available data $(n=11888)$ in the registry and therefore sample size was not undertaken. We used the Firth ${ }^{23}$ binary logistic regression model to identify factors associated with inpatient mortality. This model is an extension of the usual logistic regression model, but allows us to handle the issue of separation in the data (ie, problems with zero cell counts). For inpatient mortality, we assessed the potential interaction effects between age and the other covariates in the multivariable model based on apriori beliefs. We reported the coefficients (or ORs) and their 95\% CIs as measures of effect size. Based on the final multivariable model identified for inpatient mortality, we assessed the discriminatory properties by calculating the area under the curve (AUC). We calculated these statistics separately for the model development and validation samples to assess the robustness of our models. To evaluate the internal validity of the models, we calculated the bootstrapped (1000 replications) AUC and 95\% CI for the model for inpatient mortality.

For LOS, we used the robust regression model, with a tuning factor of 10 , which allowed us to downweigh the contribution of outliers in the model, since LOS is heavily right skewed and not normally distributed. As mortality cannot be deemed a confounder for LOS and due to the potentially mediating effect of this variable on the causal pathway, we included only survivors among predictors of LOS and undertook a sensitivity analysis where those who died were included in the analysis. For LOS, model performance was quantified by the $\mathrm{R}^{2}$ statistic. For both outcomes, we undertook univariable and multivariable analyses. Starting from the most statistically significant covariate identified in the univariable model, we used the likelihood ratio test to examine whether the inclusion of the next most significant variable helped improve the fit of the model, and this was sequentially done until all the covariates were examined.

For age, we used restricted cubic splines ${ }^{24}$ to model for non-linear relationships with outcome and to identify cutoffs where there was an inflection in the risk of outcome. The number of knots for the cubic splines were selected based on the Akaike information criterion (AIC) measure and was set at four knots at the following age values of 16 , 50,75 and 100. ISS was left as an ordinal variable in the modelling as this was deemed most clinically relevant. As ISS and AIS scores were collinear, we opted to include the more important covariate based on the AIC statistic, in the multivariable model. Missing data for each covariate were kept as a separate category in our model.

To address the potential impact of survivorship bias, that is, a different interpretation of LOS for those who are alive at discharge compared with those who have died, we undertook separate models for those who were alive versus those who died (sensitivity analysis presented in online supplemental file 1 of the manuscript). We also undertook sensitivity analysis to assess the robustness of our models, specifically by including transfer patients in our analysis. Level of significance was set at $5 \%$ and data analysed in Stata V.16 (Stata Corp, College Station, Texas, USA).

\section{RESULTS}

A total of 16965 patients sustained major trauma in Australia or New Zealand and were managed at an ATR site between July 2016 and June 2018. Of these patients, 5061 patients were transferred from another hospital, and 16 had unknown transfer status; these patients were excluded from the main analysis. Of the remaining 11888 patients, $80 \%$ (9509) were used in the model-building process and the remaining for model validation. This study was based on all available patient data, and our large sample size of 9509 (80\%) was sufficiently powered to detect small effect sizes (at least a $25 \%$ increase in odds of outcome).$^{25}$

Table 1 shows the demographic and clinical characteristics of the patients included in the model-building cohort. About half $(50.2 \%)$ were more than 50 years old at the time of the trauma event; the majority $(72.0 \%)$ were male. Motor vehicle occupant was the most common injury cause $(24.6 \%)$, followed by low fall $(17.7 \%)$ and high fall (defined as $>1 \mathrm{~m} ; 16.4 \%$ ). The median LOS was 7.7 days (IQR: 3.7-15.0). The highest AIS score was most commonly $3(51.1 \%)$, while the most common secondhighest AIS value was 2. The majority belonged to ISS group 13-22 (67.7\%) followed by 24-43 (28.8\%). There were significant differences in all variables in table 1 between those who died versus those who remained alive.

Table 2 highlights the univariable factors associated with mortality. Age defined by cubic splines, sex, ATR injury cause, arrival GCS motor score, highest and secondhighest AIS scores, HR and SBP, SI and ISS were significantly associated with inpatient mortality. Restricted cubic spline plots showed that there was a non-linear association between age and mortality (figure 1).

Compared with evaluating age as a continuous or categorical variable, we found that the cubic splines provided the best fit, with the lowest AIC value of 5825.8. Females had a $20 \%$ increased odds of mortality (95\% CI: $4 \%$ to $39 \%$ ) when compared with males, $\mathrm{p}=0.014$. A doseresponse relationship was observed for arrival GCS motor score, with those 'obeying commands' (score 6) showing a $96 \%$ decrease (95\% CI: $95 \%$ to $97 \%$ ) in odds of mortality compared with those in the 'none' category (score 1; $\mathrm{p}<0.001$ ). Similar dose-response relationships were seen for the relationship between highest as well as the second-highest AIS scores, SI and ISS with mortality.

In the multivariable analysis (table 3 ), all of the variables originally significant in the univariable analysis remained significant, except for sex. We observed that effect sizes for age group, AIS scores and SI were smaller in size, while for injury cause and GCS arrival score, the effect sizes were larger in the multivariable analysis, probably due to confounding. Due to collinearity between SI and both HR and SBP, we opted to include SI in the multivariable model, as it was found to be the stronger predictor 
Table 1 Demographic and clinical variables by inpatient mortality status

\begin{tabular}{|c|c|c|c|c|}
\hline Variables & Alive & Dead & All & $P$ value \\
\hline $\mathrm{N}$ & 8579 & 930 & 9509 & \\
\hline Age group & & & & $<0.001$ \\
\hline$<21$ & $673(7.8 \%)$ & $47(5.1 \%)$ & $720(7.6 \%)$ & \\
\hline $21-30$ & $1435(16.7 \%)$ & $113(12.2 \%)$ & $1548(16.3 \%)$ & \\
\hline $31-40$ & $1155(13.5 \%)$ & $63(6.8 \%)$ & $1218(12.8 \%)$ & \\
\hline $41-50$ & $1173(13.7 \%)$ & $74(8.0 \%)$ & $1247(13.1 \%)$ & \\
\hline $51-60$ & $1338(15.6 \%)$ & $94(10.1 \%)$ & $1432(15.1 \%)$ & \\
\hline $61-70$ & $1053(12.3 \%)$ & $123(13.2 \%)$ & $1176(12.4 \%)$ & \\
\hline $71-80$ & $895(10.4 \%)$ & $154(16.6 \%)$ & $1049(11.0 \%)$ & \\
\hline $81+$ & $854(10.0 \%)$ & $262(28.2 \%)$ & $1116(11.7 \%)$ & \\
\hline Unknown & $3(<0.1 \%)$ & $0(0.0 \%)$ & $3(<0.1 \%)$ & \\
\hline ATR injury cause & & & & $<0.001$ \\
\hline High fall & $1399(16.3 \%)$ & $163(17.5 \%)$ & $1562(16.4 \%)$ & \\
\hline Low fall & $1416(16.5 \%)$ & $268(28.8 \%)$ & $1684(17.7 \%)$ & \\
\hline Motor cyclist & $1262(14.7 \%)$ & $68(7.3 \%)$ & 1330 (14.0\%) & \\
\hline Motor vehicle occupant & $2170(25.3 \%)$ & $167(18.0 \%)$ & 2337 (24.6\%) & \\
\hline Other transport related & $187(2.2 \%)$ & $7(0.8 \%)$ & $194(2.0 \%)$ & \\
\hline Pedal cyclist & $642(7.5 \%)$ & $26(2.8 \%)$ & 668 (7.0\%) & \\
\hline Pedestrian & $637(7.4 \%)$ & $120(12.9 \%)$ & $757(8.0 \%)$ & \\
\hline Striking against or by object & $229(2.7 \%)$ & $12(1.3 \%)$ & $241(2.5 \%)$ & \\
\hline Struck by or collision with a person & $240(2.8 \%)$ & $8(0.9 \%)$ & $248(2.6 \%)$ & \\
\hline Other & $397(4.6 \%)$ & $91(9.8 \%)$ & $488(5.1 \%)$ & \\
\hline LOS, median (IQR) & $8.0(4.0-16.0)$ & $2.0(1.0-6.6)$ & $7.7(3.7-15.0)$ & $<0.001$ \\
\hline Sex & & & & 0.044 \\
\hline Male & $6210(72.4 \%)$ & $638(68.6 \%)$ & $6848(72.0 \%)$ & \\
\hline Female & 2367 (27.6\%) & 292 (31.4\%) & 2659 (28.0\%) & \\
\hline Not stated & $2(<1 \%)$ & $0(0.0 \%)$ & $2(<0.1 \%)$ & \\
\hline Arrival GCS motor & & & & $<0.001$ \\
\hline None (1) & $1145(13.3 \%)$ & $615(66.1 \%)$ & $1760(18.5 \%)$ & \\
\hline Extension to pain (2) & $16(0.2 \%)$ & $11(1.2 \%)$ & $27(0.3 \%)$ & \\
\hline Flexion to pain (3) & $28(0.3 \%)$ & $14(1.5 \%)$ & $42(0.4 \%)$ & \\
\hline Withdraws to pain (4) & $83(1.0 \%)$ & $28(3.0 \%)$ & $111(1.2 \%)$ & \\
\hline Localises pain (5) & $377(4.4 \%)$ & $70(7.5 \%)$ & $447(4.7 \%)$ & \\
\hline Obeys commands (6) & $6930(80.8 \%)$ & $192(20.6 \%)$ & 7122 (74.9\%) & \\
\hline Highest AIS score & & & & $<0.001$ \\
\hline 1 & $4(<1 \%)$ & $0(0.0 \%)$ & $4(<0.1 \%)$ & \\
\hline 2 & $81(0.9 \%)$ & $0(0.0 \%)$ & $81(0.9 \%)$ & \\
\hline 3 & $4751(55.4 \%)$ & 109 (11.7\%) & $4860(51.1 \%)$ & \\
\hline 4 & 2628 (30.6\%) & $170(18.3 \%)$ & $2798(29.4 \%)$ & \\
\hline 5 & 1103 (12.9\%) & $610(65.6 \%)$ & $1713(18.0 \%)$ & \\
\hline \multirow[t]{2}{*}{6} & $8(0.1 \%)$ & $35(3.8 \%)$ & $43(0.5 \%)$ & \\
\hline & $4(<1 \%)$ & $6(0.6 \%)$ & $10(0.1 \%)$ & \\
\hline Second-highest AIS score & & & & $<0.001$ \\
\hline 1 & $652(7.6 \%)$ & $126(13.5 \%)$ & $778(8.2 \%)$ & \\
\hline 2 & 4552 (53.1\%) & 202 (21.7\%) & 4754 (50.0\%) & \\
\hline
\end{tabular}


Table 1 Continued

\begin{tabular}{|c|c|c|c|c|}
\hline Variables & Alive & Dead & All & P value \\
\hline 3 & $2055(24.0 \%)$ & 237 (25.5\%) & $2292(24.1 \%)$ & \\
\hline 4 & $253(2.9 \%)$ & $106(11.4 \%)$ & $359(3.8 \%)$ & \\
\hline \multirow[t]{2}{*}{5} & $34(0.4 \%)$ & $61(6.6 \%)$ & $95(1.0 \%)$ & \\
\hline & $1033(12.0 \%)$ & $198(21.3 \%)$ & $1231(12.9 \%)$ & \\
\hline SBP, median (IQR) & $132.0(118.0-150.0)$ & $131.0(98.0-160.0)$ & $132.0(116.0-150.0)$ & 0.034 \\
\hline HR, median (IQR) & $84.0(71.0-98.0)$ & $88.0(70.0-112.0)$ & $84.0(71.0-99.0)$ & 0.016 \\
\hline Shock-index grouped in quartiles & & & & $<0.001$ \\
\hline First quartile & $2058(24.0 \%)$ & $234(25.2 \%)$ & $2292(24.1 \%)$ & \\
\hline Second quartile & $2313(27.0 \%)$ & $131(14.1 \%)$ & $2444(25.7 \%)$ & \\
\hline Third quartile & $2039(23.8 \%)$ & $138(14.8 \%)$ & $2177(22.9 \%)$ & \\
\hline Fourth quartile & $1980(23.1 \%)$ & $285(30.6 \%)$ & 2265 (23.8\%) & \\
\hline Missing & $187(2.2 \%)$ & $71(7.6 \%)$ & $258(2.7 \%)$ & \\
\hline Cardiac arrest on arrival & $2(<0.1 \%)$ & $71(7.6 \%)$ & $73(0.8 \%)$ & \\
\hline ISS grouped & & & & $<0.001$ \\
\hline $13-22$ & $6262(73.0 \%)$ & $177(19.0 \%)$ & 6439 (67.7\%) & \\
\hline $24-43$ & 2147 (25.0\%) & 591 (63.5\%) & 2738 (28.8\%) & \\
\hline $45-51$ & $112(1.3 \%)$ & 73 (7.8\%) & 185 (1.9\%) & \\
\hline $54-59$ & $36(0.4 \%)$ & $28(3.0 \%)$ & $64(0.7 \%)$ & \\
\hline $66-75$ & $22(0.3 \%)$ & 61 (6.6\%) & 83 (0.9\%) & \\
\hline
\end{tabular}

AIS, Abbreviated Injury Scale; ATR, Australian Trauma Registry; GCS, Glasgow Coma Scale; HR, heart rate; ISS, Injury Severity Score; LOS, length of stay; SBP, systolic blood pressure.

among the variables. Similarly, AIS scores were included in the model instead of ISS scores due to collinearity. We also found that those with missing SI or cardiac arrest on arrival had a higher OR of mortality of 2.2 (95\% CI: 1.4 to 3.5), $\mathrm{p}=0.001$ and 88.9 (95\% CI: 23.3 to 339.2 ), $\mathrm{p}<0.001$ as compared with those in the first quartile.

The final multivariable model returned an AUC of 0.938 (95\% CI: 0.931 to 0.945 ) (figure 2A). The bootstrapped $95 \%$ CI was reasonably narrow with a range of $0.932-0.945$. In the validation dataset, the model also performed very well with an AUC of 0.931 (95\% CI: 0.930 to 0.943 ) (figure 2B). There was no significant interaction between age and any of the other variables in the multivariable model.

Table 4 highlights univariable factors associated with LOS among patients with trauma who survived. We found age, ATR injury cause, arrival GCS motor score, highest and second-highest AIS scores, HR, SBP, SI and ISS score were all significantly associated with LOS. There was a significant dose-response relationship between arrival GCS motor score and LOS, where for example those who 'obey commands' (score 6) stayed on average 11.6 days less (95\% CI: 11.0 to 12.2 days) than those who were recorded as 'none', (score $1 ; \mathrm{p}<0.001$ ). Compared with low falls, 'motor vehicle occupants' and pedestrians stayed longer in hospital. A dose-response relationship was also observed with highest and second-highest AIS scores, where patients with higher scores stayed longer.
Patients belonging to the fourth quartile of the SI stayed on average 5.1 days (95\% CI: 4.6 to 5.6 days) more than those in the first quartile, and this was statistically significant $(p<0.001)$. Except for ISS=32 and 42, higher ISS values were associated with a longer hospital stay.

All of the associations found in the univariable analysis persisted in the multivariable analysis (table 5). The nonlinear relationship with age remained, while the coefficients changed for various age groups. The coefficient for 'cubic spline among those aged 16-50' also changed in direction. Motor vehicle occupants stayed on average 2.6 days (95\% CI: 1.3 to 2.6) longer than those who had low falls, $\mathrm{p}<0.001$. Those who exhibited withdrawal to pain, localised pain and obeying commands stayed on average for a shorter LOS as compared with those who had 'none' for arrival GCS score. Generally, those with higher SI stayed longer in hospital, while those who had a cardiac arrest on arrival stayed for a much shorter duration $(-14.7$ days $), p=0.004$. Those with higher AIS scores stayed longer and those with just one AIS score (ie, coded "Not Applicable" NA for second-highest AIS) stayed on average 4.5 days less (95\% CI: 3.9 to 5.1 days lesser) compared with those who scored 3 for the second-highest AIS, $p<0.001$. Based on the final multivariable model, we found that the model explained $13.1 \%$ of the variation in LOS. The model performed similarly well for the validation cohort, with $17.1 \%$ of the variation in LOS explained by the model. 
Table 2 Univariable factors associated with mortality

\begin{tabular}{lllll}
\hline Variable & OR & $\mathbf{9 5 \%} \mathbf{C l}$ & & P value \\
\hline Age* $^{*}$ & & & & \\
$\quad$ Cubic spline among those aged $16-50$ & 0.98 & 0.96 & 0.99 & 0.001 \\
Cubic spline among those aged $51-75$ & 1.10 & 1.06 & 1.14 & $<0.001$ \\
Cubic spline among those aged $>75$ & 0.77 & 0.67 & 0.90 & 0.001
\end{tabular}

\section{Sex}

Male

Reference

$\begin{array}{lllll}\text { Female } & 1.20 & 1.04 & 1.39 & 0.014 \\ \text { Not stated } & 1.95 & 0.09 & 40.56 & 0.668\end{array}$

ATR injury cause

$\begin{array}{lllll}\text { High fall } & 0.62 & 0.50 & 0.76 & <0.001 \\ \text { Low fall } & \text { Reference } & & & <0.001 \\ \text { Motor cyclist } & 0.29 & 0.22 & 0.38 & <0.001 \\ \text { Motor vehicle occupant } & 0.41 & 0.33 & 0.50 & 0.146 \\ \text { Other } & 1.21 & 0.93 & 1.58 & <0.001 \\ \text { Other transport related } & 0.21 & 0.10 & 0.44 & <0.001 \\ \text { Pedal cyclist } & 0.22 & 0.14 & 0.33 & 0.981 \\ \text { Pedestrian } & 1.00 & 0.79 & 1.26 & <0.001 \\ \text { Striking against or by object } & 0.29 & 0.16 & 0.52 & <0.001\end{array}$

Arrival GCS motor

\begin{tabular}{|c|c|c|c|c|}
\hline \multirow{2}{*}{$\begin{array}{l}\text { None (1) } \\
\text { Extension to pain (2) }\end{array}$} & \multicolumn{4}{|c|}{ Reference } \\
\hline & 1.07 & 0.51 & 2.26 & 0.853 \\
\hline Flexion to pain (3) & 0.74 & 0.40 & 1.36 & 0.333 \\
\hline Withdraws to pain (4) & 0.47 & 0.30 & 0.72 & 0.001 \\
\hline Localises pain (5) & 0.27 & 0.21 & 0.35 & $<0.001$ \\
\hline Obeys commands (6) & 0.04 & 0.03 & 0.05 & $<0.001$ \\
\hline Not stated & 0.24 & 0.18 & 0.32 & $<0.001$ \\
\hline \multicolumn{5}{|l|}{ Highest AIS score } \\
\hline 1 & 4.82 & 0.26 & 90.10 & 0.292 \\
\hline 2 & 0.27 & 0.02 & 4.32 & 0.352 \\
\hline 3 & \multicolumn{4}{|c|}{ Reference } \\
\hline 4 & 2.81 & 2.20 & 3.60 & $<0.001$ \\
\hline 5 & 24.01 & 19.39 & 29.73 & $<0.001$ \\
\hline 6 & 181.23 & 83.74 & 392.21 & $<0.001$ \\
\hline \multicolumn{5}{|l|}{ Second-highest AIS score } \\
\hline 1 & 1.68 & 1.33 & 2.12 & $<0.001$ \\
\hline 2 & 0.38 & 0.32 & 0.47 & $<0.001$ \\
\hline 3 & \multicolumn{4}{|c|}{ Reference } \\
\hline 4 & 3.64 & 2.79 & 4.73 & $<0.001$ \\
\hline 5 & 15.43 & 9.96 & 23.91 & $<0.001$ \\
\hline NA & 1.66 & 1.36 & 2.04 & $<0.001$ \\
\hline SBP & 0.99 & 0.99 & 0.99 & $<0.001$ \\
\hline HR & 1.00 & 1.00 & 1.01 & 0.033 \\
\hline \multicolumn{5}{|c|}{ Shock-index grouped in quartiles } \\
\hline First quartile & \multicolumn{4}{|c|}{ Reference } \\
\hline
\end{tabular}

Continued 


\begin{tabular}{|c|c|c|c|c|}
\hline Variable & OR & $95 \% \mathrm{Cl}$ & & $P$ value \\
\hline Second quartile & 0.50 & 0.40 & 0.62 & $<0.001$ \\
\hline Third quartile & 0.60 & 0.48 & 0.74 & $<0.001$ \\
\hline Fourth quartile & 1.27 & 1.05 & 1.52 & 0.012 \\
\hline Missing & 3.35 & 2.47 & 4.54 & $<0.001$ \\
\hline Cardiac arrest on arrival & 251.06 & 70.63 & 892.47 & $<0.001$ \\
\hline \multicolumn{5}{|l|}{ ISS } \\
\hline 13 & \multicolumn{4}{|c|}{ Reference } \\
\hline 14 & 0.96 & 0.50 & 1.83 & 0.899 \\
\hline 16 & 3.97 & 2.16 & 7.32 & $<0.001$ \\
\hline 17 & 2.33 & 1.34 & 4.07 & 0.003 \\
\hline 18 & 2.99 & 1.36 & 6.56 & 0.006 \\
\hline 19 & 2.68 & 1.22 & 5.88 & 0.014 \\
\hline 20 & 3.11 & 1.38 & 7.00 & 0.006 \\
\hline 21 & 2.47 & 1.15 & 5.30 & 0.020 \\
\hline 22 & 2.22 & 1.13 & 4.34 & 0.020 \\
\hline 24 & 3.18 & 1.53 & 6.61 & 0.002 \\
\hline 25 & 28.41 & 16.76 & 48.17 & $<0.001$ \\
\hline 26 & 29.16 & 17.23 & 49.37 & $<0.001$ \\
\hline 27 & 8.83 & 4.45 & 17.54 & $<0.001$ \\
\hline 29 & 12.21 & 7.05 & 21.14 & $<0.001$ \\
\hline 30 & 28.07 & 15.62 & 50.47 & $<0.001$ \\
\hline 32 & 10.66 & 1.78 & 63.83 & 0.010 \\
\hline 33 & 14.91 & 7.80 & 28.51 & $<0.001$ \\
\hline 34 & 13.60 & 7.30 & 25.34 & $<0.001$ \\
\hline 35 & 31.92 & 15.74 & 64.70 & $<0.001$ \\
\hline 36 & 5.68 & 1.93 & 16.69 & 0.002 \\
\hline 38 & 32.20 & 18.03 & 57.53 & $<0.001$ \\
\hline 41 & 13.51 & 6.08 & 30.01 & $<0.001$ \\
\hline 42 & 58.54 & 18.40 & 186.24 & $<0.001$ \\
\hline 43 & 38.71 & 19.45 & 77.01 & $<0.001$ \\
\hline 45 & 42.29 & 22.08 & 81.03 & $<0.001$ \\
\hline 48 & 8.81 & 1.50 & 51.70 & 0.016 \\
\hline 50 & 51.57 & 26.83 & 99.13 & $<0.001$ \\
\hline 51 & 121.58 & 24.05 & 614.76 & $<0.001$ \\
\hline 54 & 94.56 & 32.91 & 271.75 & $<0.001$ \\
\hline 57 & 39.83 & 16.59 & 95.65 & $<0.001$ \\
\hline 59 & 48.25 & 16.79 & 138.65 & $<0.001$ \\
\hline 66 & 77.55 & 32.27 & 186.36 & $<0.001$ \\
\hline 75 & 330.62 & 141.25 & 773.86 & $<0.001$ \\
\hline
\end{tabular}

${ }^{\star}$ No reference category exists for age as the cubic splines represent non-linear relationships; resultant ORs are not easily interpretable. AIS, Abbreviated Injury Scale; ATR, Australian Trauma Registry; GCS, Glasgow Coma Scale; HR, heart rate; ISS, Injury Severity Score; LOS, length of stay; SBP, systolic blood pressure.

\section{Sensitivity analysis}

We undertook a number of sensitivity analyses to test the robustness of our models against various assumptions.
First, we assessed the impact of including transferred patients on our multivariable results. We found that the main implication was that the coefficients for several 


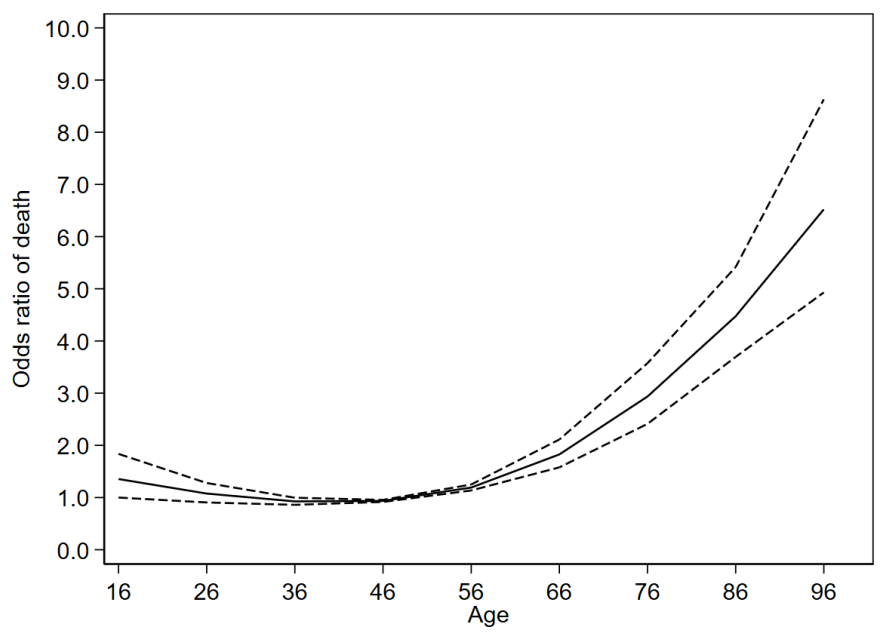

Figure 1 Restricted cubic splines for relationship between age and inpatient mortality. 95\% Cls are shown as dashed lines.

of the covariates for the mortality outcome were less pronounced when we included the transfers (online supplemental table S1). For example, motor vehicle occupant injury cause and a highest AIS score of 1 were no longer significant, whereas for some covariates, the effects were attenuated such as pedal cyclist (OR changed from 0.66 to 0.39 and became significant, $p=0.013$ ). In particular, we noted substantial changes in the magnitude and significance for SI, which became more pronounced when the transfers were included. The results potentially indicate a marked increase in effect size due to the inclusion of sicker patients but independent of other measures of severity (ie, highest AIS, GCS motor).

As for LOS, there were no materially significant changes to the magnitude or direction of the effect sizes, except for the group with an AIS score of 6, where we found there was an increase in LOS from 8.2 days to 12.7 days, potentially caused by the more ill patients included in our analysis, but the numbers are small in this group (online supplemental table S2).

Online supplemental table S3 shows the results for the multivariable model for LOS including those who died. When deaths were included in the model (online supplemental table 3), the following differences to the multivariable results for LOS were observed: pedal cyclists and those 'struck by or collision with a person' which were originally not significant became statistically significant, staying 1.3 and 1.2 days less, respectively compared with those who had a 'low fall'. Arrival GCS motor score was also not as important with only two categories, 'localises pain' and 'obeys commands' remaining significant. Similarly, the magnitude of the effect sizes was reduced for AIS scores and those with cardiac arrest on arrival; there was a change in the direction of effect for the highest AIS score (6) from +8.2 days to -3.8 days, and the second-highest AIS score (5) from 10.8 days to -3.7 days compared with the reference of AIS 3. Given that patients with AIS 5 or 6 injuries are more likely to die, often following comparatively little time in hospital, this finding is unsurprising.
Table 3 Multivariable factors associated with mortality

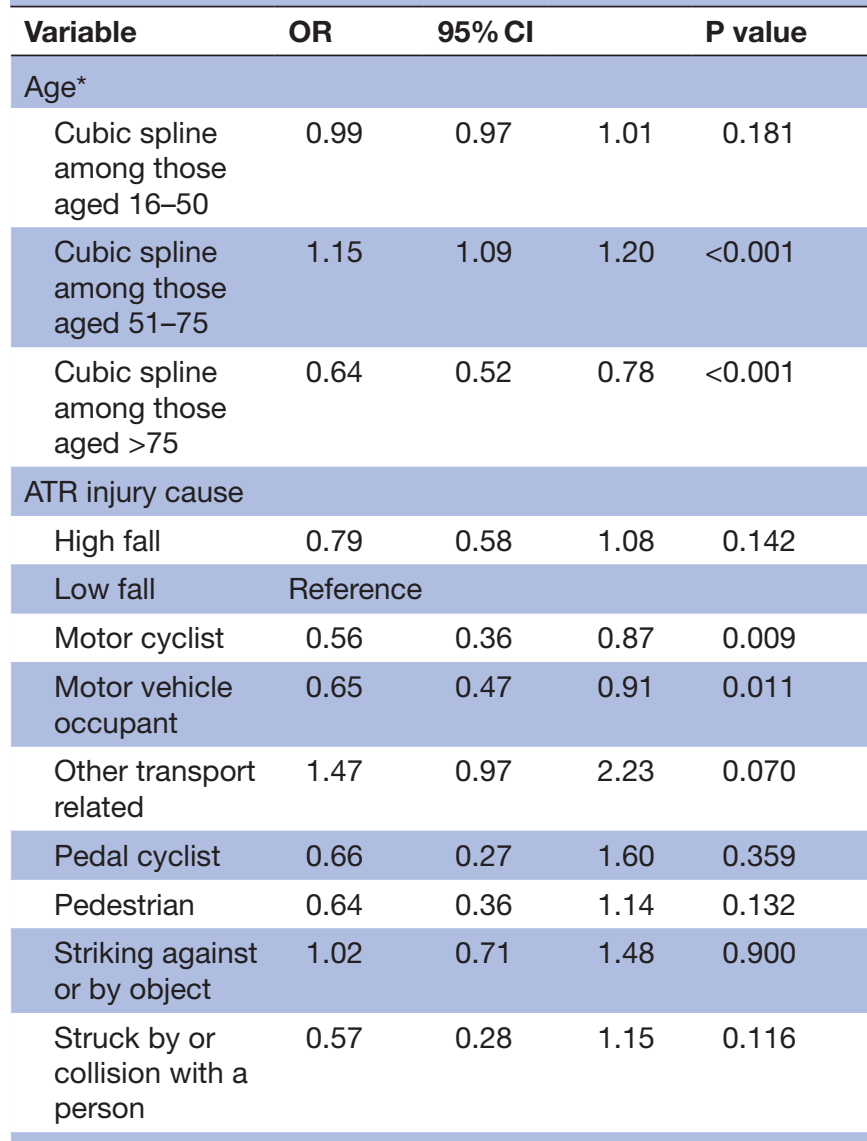

$\begin{array}{ccccc}\text { Other } & 0.56 & 0.24 & 1.31 & 0.185 \\ \text { Arrival GCS motor } & & & & \end{array}$

\begin{tabular}{|c|c|c|c|c|}
\hline None (1) & \multicolumn{4}{|c|}{ Reference } \\
\hline $\begin{array}{l}\text { Extension to } \\
\text { pain (2) }\end{array}$ & 0.52 & 0.19 & 1.42 & 0.205 \\
\hline $\begin{array}{l}\text { Flexion to pain } \\
\text { (3) }\end{array}$ & 0.52 & 0.23 & 1.17 & 0.113 \\
\hline $\begin{array}{l}\text { Withdraws to } \\
\text { pain (4) }\end{array}$ & 0.28 & 0.16 & 0.50 & $<0.001$ \\
\hline $\begin{array}{l}\text { Localises pain } \\
\text { (5) }\end{array}$ & 0.17 & 0.12 & 0.25 & $<0.001$ \\
\hline $\begin{array}{l}\text { Obeys } \\
\text { commands (6) }\end{array}$ & 0.04 & 0.03 & 0.05 & $<0.001$ \\
\hline Not stated & 0.15 & 0.10 & 0.23 & $<0.001$ \\
\hline \multicolumn{5}{|l|}{ Highest AIS score } \\
\hline 1 & 34.33 & 1.78 & 662.40 & 0.019 \\
\hline 2 & 0.15 & 0.01 & 2.69 & 0.195 \\
\hline 3 & \multicolumn{4}{|c|}{ Reference } \\
\hline 4 & 1.69 & 1.26 & 2.28 & $<0.001$ \\
\hline 5 & 8.12 & 6.12 & 10.78 & $<0.001$ \\
\hline 6 & 37.60 & 12.89 & 109.64 & $<0.001$ \\
\hline \multicolumn{5}{|c|}{ Second-highest AIS score } \\
\hline 1 & 0.86 & 0.61 & 1.19 & 0.358 \\
\hline 2 & 0.60 & 0.46 & 0.78 & $<0.001$ \\
\hline 3 & \multicolumn{4}{|c|}{ Reference } \\
\hline
\end{tabular}

Continued 


\begin{tabular}{ccccc}
\hline \multicolumn{2}{l}{ Table 3 } & \multicolumn{2}{l}{ Continued } \\
\hline Variable & OR & $\mathbf{9 5 \%} \mathbf{C l}$ & & P value \\
\hline 4 & 0.99 & 0.69 & 1.42 & 0.946 \\
5 & 1.76 & 1.02 & 3.04 & 0.044 \\
NA & 0.71 & 0.52 & 0.98 & 0.038
\end{tabular}

Shock-index grouped in quartiles

\begin{tabular}{lcrrr} 
First quartile & Reference & & & \\
Second quartile & 0.68 & 0.52 & 0.91 & 0.008 \\
Third quartile & 0.96 & 0.72 & 1.27 & 0.754 \\
Fourth quartile & 1.09 & 0.83 & 1.44 & 0.517 \\
Missing & 2.20 & 1.39 & 3.48 & 0.001 \\
$\begin{array}{l}\text { Cardiac arrest } \\
\text { on arrival }\end{array}$ & 88.92 & 23.31 & 339.16 & $<0.001$ \\
\hline
\end{tabular}

${ }^{*}$ No reference category exists for age as the cubic splines represent non-linear relationships; resultant ORs are not easily interpretable.

AIS, Abbreviated Injury Scale; ATR, Australian Trauma Registry; GCS, Glasgow Coma Scale.

\section{DISCUSSION}

Trauma is a time critical disease, ${ }^{26}$ and effective management across trauma centres and trauma systems can measurably affect outcomes. Many different approaches have been developed and evolved to benchmark trauma care over time, but different models have resulted in different conclusions being drawn. Using contemporary statistical techniques, this study has found that the important factors associated with trauma inpatient mortality include age group, gender, cause of injury, arrival GCS motor score, highest and second-highest AIS score, SI group and ISS group. The overall model which was developed performed well, with an AUC of 0.938. As for LOS, the same factors (except for sex) were found to be associated in the multivariable analysis. These findings
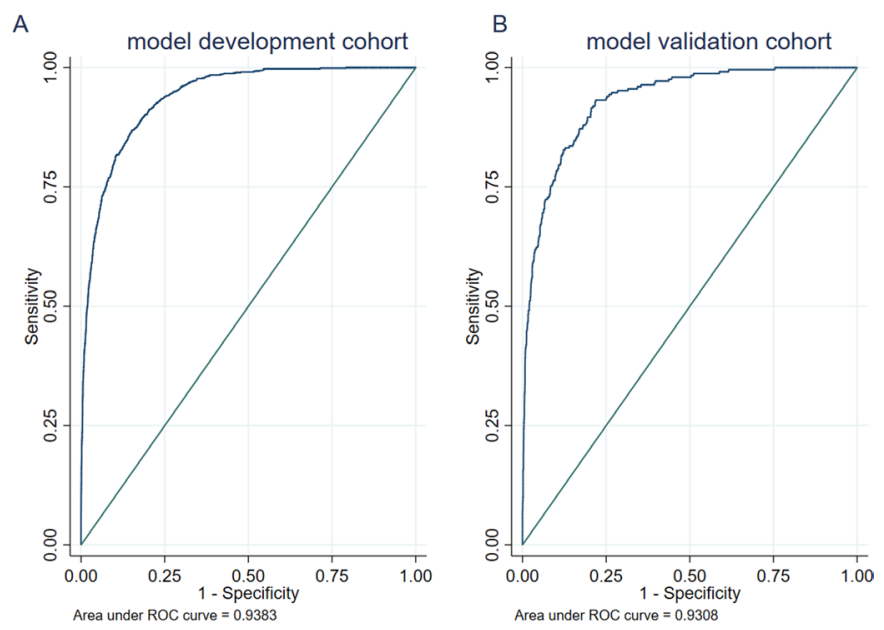

Figure 2 Area under the curve for the final multivariable model for mortality. (A) Model development cohort and (B) Model validation cohort. ROC:Receiver Operating Characteristic will allow us to provide for transparent and robust benchmarking of outcomes among trauma centres. The results could also potentially aid clinicians to risk stratify patients when they present to the hospital.

Our model performance (AUC=0.938) was similar to a recently published model RISC II $(\mathrm{AUC}=0.953){ }^{8}$ Importantly, our model is based on a smaller number of variables, that are more readily available for trauma registries to collect. This small number of variables allows less chance of measurement error and bias and greater feasibility of collection. In addition, the model deals with missing data using a single unified statistical approach, without making assumptions which are difficult to ascertain. The RISC II model has 15 variables (3 derived from AIS), while the model developed in this study is based on just 6 variables. Some of the laboratory variables included in the RISC II model could also be prone to incomplete data collection or missing data.

As with most observational studies, missing data are of concern and there are several techniques to deal with the problem, including complete case analysis, single imputation and multiple imputation. Imputing data would require the assumption that the data are missing at random, which could be problematic in the context of data collected from sites for the purposes of benchmarking. Therefore, we did not undertake multiple imputation of missing data. Similarly, we randomly selected $20 \%$ of the observations across the entire study period to perform the validation exercise and not by calendar year as we observed differential levels of missing data by site during the more recent data collection period.

The approach to missing data in the RISC II model involves assigning them to existing patient categories which are more 'similar' to them in terms of risk of outcome and assigning the group as the reference category. This two-stage process does not account for uncertainty adequately in the modelling process. Such an approach is also data driven and not easily scalable to other population or datasets. For instance, in the RISC II model, missing data in the highest AIS score category is assigned to 2, and in the second-highest AIS score category to 3 ; this is not clinically intuitive.

There is also a similar risk-adjustment work undertaken by the American College of Surgeons' (ACS) Trauma Quality Improvement Program (TQIP). ${ }^{27}$ There are a few major differences between their approach and ours. First, the ACS' TQIP considers additional variables such as individual comorbidities, race and payment type, which are not readily available in our registry. Second, they have included hospital effects as a random intercept term within a hierarchical regression model. Such an approach 'can inadvertently 'adjust away' some of the key inter-hospital process and outcomes differences that are the focus of TQIP' as acknowledged by the same authors. While including hospital effects may help with a patientrisk stratification model, we feel it may not be appropriate for a benchmarking exercise. 
Table 4 Univariable factors associated with length of stay among survivors

\begin{tabular}{|c|c|c|c|c|}
\hline Variable & Coefficient & $95 \% \mathrm{Cl}$ & & $P$ value \\
\hline \multicolumn{5}{|l|}{ Age $^{*}$} \\
\hline Cubic spline among those aged $16-50$ & -0.05 & -0.08 & -0.02 & 0.004 \\
\hline Cubic spline among those aged 51-75 & 0.16 & 0.07 & 0.25 & $<0.001$ \\
\hline Cubic spline among those aged $>75$ & -0.73 & -1.15 & -0.30 & 0.001 \\
\hline
\end{tabular}

\section{Sex}

Male

Reference

Female

Not stated

0.65

0.23

1.06

0.002

ATR injury cause

High fall

$-7.30$

$-19.26$

4.66

0.232

Low fall

0.77

0.15

1.40

0.015

Motor cyclist

Reference

Motor vehicle occupant

1.39

0.74

2.03

$<0.001$

Other

3.07

2.51

0.72

$-0.22$

3.63

$<0.001$

Other transport related

$-0.60$

$-1.89$

1.66

0.131

Pedal cyclist

$-2.31$

$-3.10$

0.70

0.367

Pedestrian

3.38

2.59

$-1.52$

$<0.001$

Striking against or by object

$-0.60$

$-1.79$

4.17

$<0.001$

Struck by or collision with a person

$-2.41$

$-3.57$

0.60

0.327

Arrival GCS motor

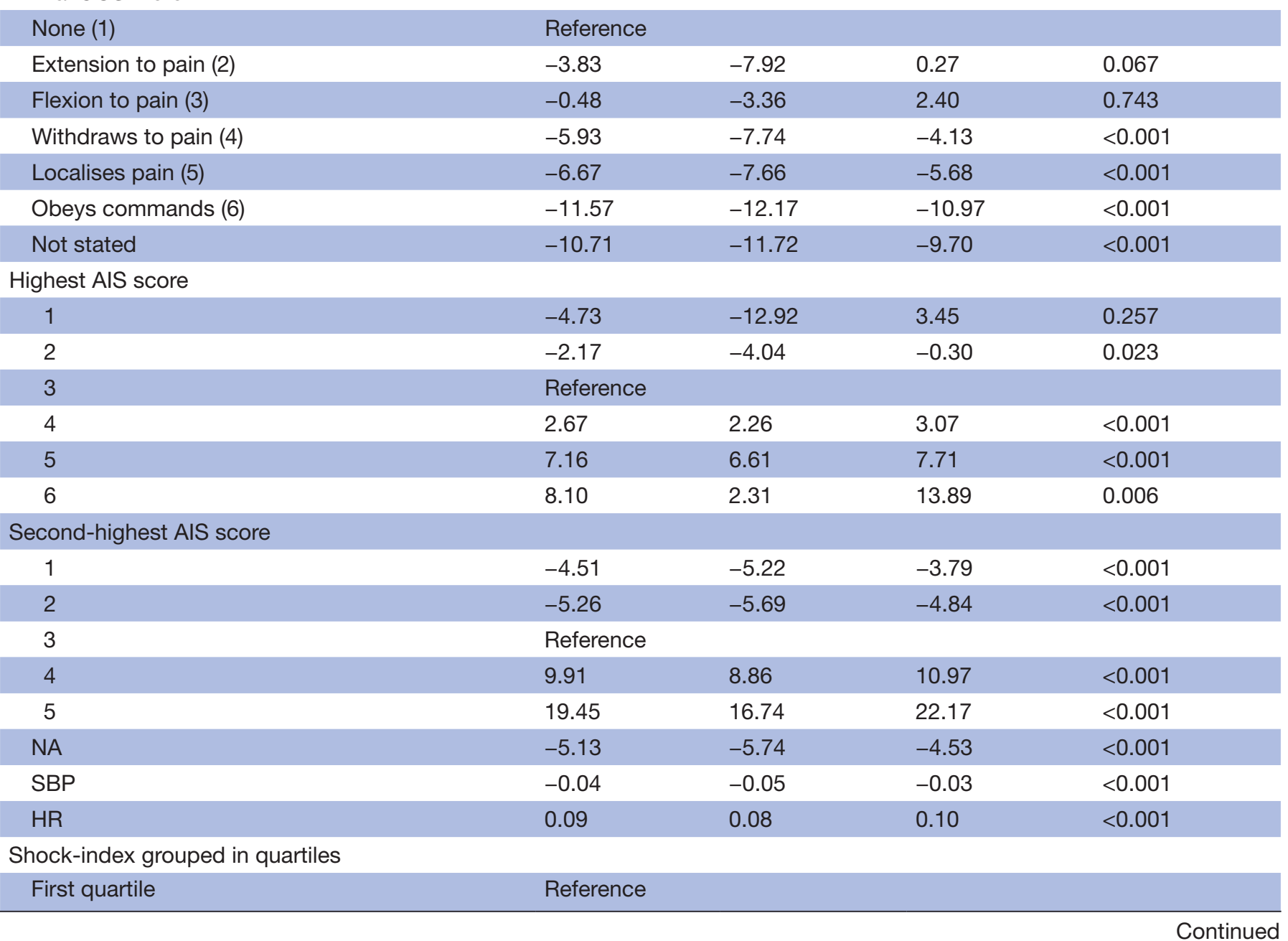

Continued 


\begin{tabular}{|c|c|c|c|c|}
\hline Variable & Coefficient & $95 \% \mathrm{Cl}$ & & $P$ value \\
\hline Second quartile & 0.22 & -0.28 & 0.73 & 0.386 \\
\hline Third quartile & 0.90 & 0.37 & 1.42 & 0.001 \\
\hline Fourth quartile & 5.08 & 4.55 & 5.60 & $<0.001$ \\
\hline Missing & 0.61 & -0.66 & 1.88 & 0.347 \\
\hline Cardiac arrest on arrival & -7.50 & -19.20 & 4.20 & 0.209 \\
\hline \multicolumn{5}{|l|}{ ISS } \\
\hline 13 & Reference & & & \\
\hline 14 & 0.11 & -0.48 & 0.71 & 0.709 \\
\hline 16 & 0.81 & 0.00 & 1.62 & 0.049 \\
\hline 17 & 1.86 & 1.27 & 2.45 & $<0.001$ \\
\hline 18 & 1.86 & 0.78 & 2.94 & 0.001 \\
\hline 19 & 2.37 & 1.33 & 3.41 & $<0.001$ \\
\hline 20 & 3.06 & 1.92 & 4.19 & $<0.001$ \\
\hline 21 & 2.74 & 1.78 & 3.70 & $<0.001$ \\
\hline 22 & 5.60 & 4.82 & 6.38 & $<0.001$ \\
\hline 24 & 6.18 & 5.18 & 7.17 & $<0.001$ \\
\hline 25 & 4.63 & 3.67 & 5.60 & $<0.001$ \\
\hline 26 & 5.34 & 4.37 & 6.31 & $<0.001$ \\
\hline 27 & 9.97 & 8.64 & 11.31 & $<0.001$ \\
\hline 29 & 7.94 & 7.05 & 8.83 & $<0.001$ \\
\hline 30 & 5.99 & 4.56 & 7.41 & $<0.001$ \\
\hline 32 & 2.60 & -2.67 & 7.87 & 0.333 \\
\hline 33 & 10.41 & 8.95 & 11.87 & $<0.001$ \\
\hline 34 & 11.20 & 9.89 & 12.50 & $<0.001$ \\
\hline 35 & 9.84 & 7.58 & 12.10 & $<0.001$ \\
\hline 36 & 13.87 & 11.70 & 16.04 & $<0.001$ \\
\hline 38 & 11.03 & 9.56 & 12.49 & $<0.001$ \\
\hline 41 & 17.70 & 15.68 & 19.72 & $<0.001$ \\
\hline 42 & 4.81 & -0.82 & 10.44 & 0.094 \\
\hline 43 & 18.57 & 16.29 & 20.86 & $<0.001$ \\
\hline 45 & 15.67 & 13.56 & 17.78 & $<0.001$ \\
\hline 48 & 26.24 & 21.74 & 30.74 & $<0.001$ \\
\hline 50 & 18.54 & 16.28 & 20.80 & $<0.001$ \\
\hline 51 & 22.47 & 11.96 & 32.98 & $<0.001$ \\
\hline 54 & 32.28 & 26.65 & 37.91 & $<0.001$ \\
\hline 57 & 21.23 & 17.71 & 24.76 & $<0.001$ \\
\hline 59 & 23.34 & 18.63 & 28.06 & $<0.001$ \\
\hline 66 & 23.91 & 19.60 & 28.22 & $<0.001$ \\
\hline 75 & 10.91 & 5.94 & 15.88 & $<0.001$ \\
\hline
\end{tabular}

${ }^{*}$ No reference category exists for age as the cubic splines represent non-linear relationships; resultant ORs are not easily interpretable. AIS, Abbreviated Injury Scale; ATR, Australian Trauma Registry; GCS, Glasgow Coma Scale; HR, heart rate; ISS, Injury Severity Score; SBP, systolic blood pressure.

A formal comparison with the various models would be problematic for several reasons. The characteristics of the study population are different and the way the variables are defined and collected could differ by countries. ${ }^{28}$ Also, the fact that the RISC II study included penetrating injuries, while we excluded them in our analysis would 
Open access

Table 5 Multivariable factors associated with length of stay among survivors

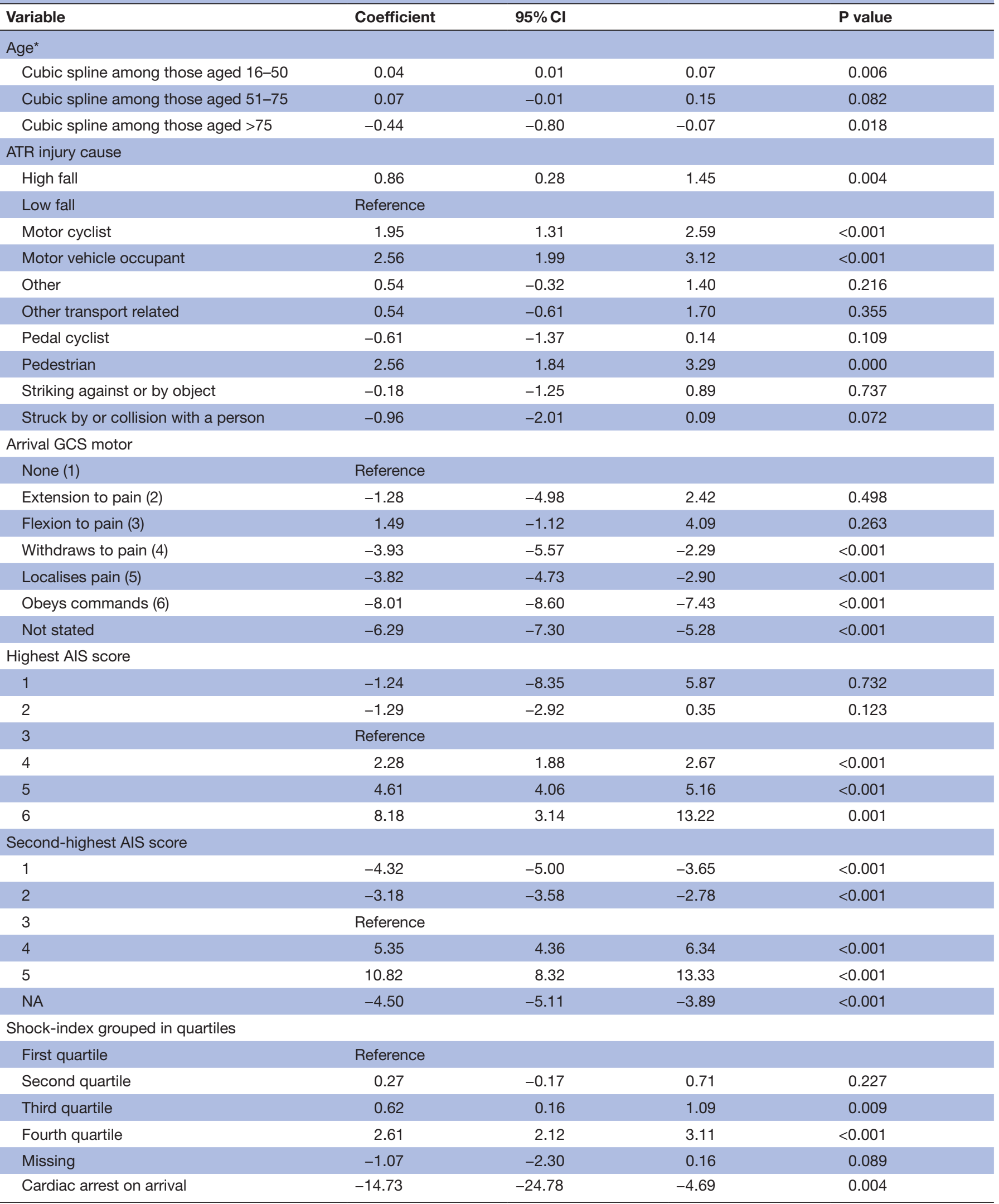

${ }^{*}$ No reference category exists for age as the cubic splines represent non-linear relationships; resultant ORs are not easily interpretable. AIS, Abbreviated Injury Scale; ATR, Australian Trauma Registry; GCS, Glasgow Coma Scale. 
preclude such a comparative analysis. The ACS' TQIP also had different inclusion/exclusion criteria from our study. As the RISC II study has already established superior performance to previous models, ${ }^{8}$ we did not repeat these comparisons in our study. Any formal comparison between the models/approaches would require simulation studies (to ensure they are valid across a variety of trauma system settings) as well as application to a dataset with broader list of variables such as the one from ACS's TQIP.

Sensitivity analyses indicated that our results were not materially altered by including transferred patients, except for differences that have been discussed earlier. As a sensitivity analysis, we also provided a model excluding deaths in the multivariable model because LOS has a different interpretation for those who died compared with those alive and it also cannot strictly be considered as a confounding variable (online supplemental table S3).

We used a number of statistical models and techniques to address the inherent limitations of large observational registries such as ours. The Firth logistic regression, which uses penalised maximum likelihood estimation was used to analyse inpatient mortality. This model is able to handle the 'separation' issue, specifically observations being dropped due to sparseness in the data. For LOS which was heavily skewed, we used the robust linear regression model, which reduces skewness based on the median absolute deviation from the median residual. ${ }^{29}$ Alternative approaches include a logarithmic transformation followed by ordinary least squares regression model, which would make the interpretation of the coefficients cumbersome and the non-parametric quantile (median) regression method, which is statistically less efficient.

SI was also included in the multivariable model rather than its component HR and SBP due to collinearity and the fact that SI was a better predictor. Similarly, ISS and AIS scores were deemed to be clinically collinear and we included AIS scores (specifically highest and secondhighest scores) as they indicated a better fit as compared with ISS (AIC=4525 and 4660 respectively).

Since our study was designed to identify risk factors associated with inpatient mortality and LOS among patients with trauma for benchmarking purposes, we did not include the centre variable in the model. Undertaking such an analysis may provide for over shrinkage in the risk-adjusted mortality rates and increase false-negative rates in terms of identifying potential outliers. One paper which attempted to use a hierarchical logistic regression model ended up shrinking the variability in the mortality rates ranging from $1.3 \%$ to $14.3 \%$ in the crude model to $3.7 \%$ to $6.9 \%$ multivariable model, thus only identifying 3 out of 59 hospitals as potential outliers in their analysis. ${ }^{30}$ The main purpose of our proposed risk-adjustment model is for benchmarking trauma centres and not predictive modelling. It is not intended to be prospectively used for system or patient-level prediction / triage. Hence a score that relies on discharge confirmation of AIS (rather than AIS at admission) is appropriate for our study.
Unmeasured confounders could affect the results of our study. For example, assessments of patient preinjury comorbidity such as the Charlson Comorbidity Index have been postulated to be associated with inpatient mortality among patients with trauma. However, this often depends on comprehensive ICD coding, which may not be routinely done; in addition the depth of trauma coding may not be consistently performed in all trauma centres. ${ }^{31}$ We also did not include laboratory variables such as International Normalised Ratio, haemoglobin and base deficit as used in other published risk models, but note that the incremental value of including these variables is marginal at the expense of a more parsimonious model with less potential for measurement error. ${ }^{8}$ Other unmeasured factors could also affect LOS, including availability and accessibility of postacute care placement services and also social factors such as availability of caregivers, family members, etc, but these were not collected within our registry.

A major strength of this study is the large sample size with data from 31 major trauma centres from across Australia and New Zealand. This large study size allows us to perform model development and validation and also undertake important sensitivity analyses around including transfer cases and excluding mortality in the multivariable analysis for LOS.

\section{CONCLUSION}

This study has identified several demographic and clinical risk factors for inpatient mortality and LOS among patients with trauma through a robust process of model development and validation. These results can be used to benchmark clinical outcomes for trauma centres employing a transparent and reproducible methodology.

\section{Author affiliations}

${ }^{1}$ Department of Epidemiology and Preventive Medicine, Monash University School of Public Health and Preventive Medicine, Melbourne, Victoria, Australia

${ }^{2}$ Trauma Service, Royal Children's Hospital Melbourne, Parkville, Victoria, Australia ${ }^{3}$ Emergency and Trauma Centre, The Alfred, Melbourne, Victoria, Australia ${ }^{4}$ National Trauma Research Institute, The Alfred, Melbourne, Victoria, Australia ${ }^{5}$ State Trauma Unit, Royal Perth Hospital, Perth, Western Australia, Australia ${ }^{6}$ Sydney Nursing School, University of Sydney, Sydney, New South Wales, Australia ${ }^{7}$ Illawarra Shoalhaven, Local Health District, Sydney, New South Wales, Australia

Acknowledgements The authors would like to thank the individual trauma centres for providing data for this study.

Contributors $A E$ conceived the study, analysed and wrote the initial draft as well as the final manuscript. CP, GO'R, MB, SR and KC provided critical input in the design of the study and writing the manuscript. EM collated the dataset and provided critical input in the design of the study and writing the manuscript. PC conceived the study and provided critical input in the design of the study and writing the manuscript.

Funding The authors have not declared a specific grant for this research from any funding agency in the public, commercial or not-for-profit sectors.

Disclaimer The funders had no role in the design, analysis and reporting of this study. Award/grant number is not applicable.

Competing interests Associate Professor GO'R is currently a NHMRC Research Fellow at the National Trauma Research Institute, Alfred Hospital, Melbourne, Australia, leading the project titled Maximising the usefulness and timeliness of 
trauma and emergency registry data for improving patient outcomes. Professor PC is funded by the Medical Research Futures Fund as a Practitioner Fellow.

\section{Patient consent for publication Not required.}

Ethics approval Each hospital has individual ethics approval for data collection from admitted patients under waiver of consent and deidentified data were sent to the national Australian Trauma Registry (ATR), which was analysed in our study. Ethics approval for this study was provided by the Alfred Hospital Ethics committee, as the Alfred Hospital is the host of the ATR database (Project No: 65/20).

Provenance and peer review Not commissioned; externally peer reviewed.

Data availability statement Data are available upon reasonable request. The Australian Trauma Registry collates data from secondary sources - either institutional trauma registries or state based registries. Data may be made available to researchers provided ethics approval is sought.

Supplemental material This content has been supplied by the author(s). It has not been vetted by BMJ Publishing Group Limited (BMJ) and may not have been peer-reviewed. Any opinions or recommendations discussed are solely those of the author(s) and are not endorsed by BMJ. BMJ disclaims all liability and responsibility arising from any reliance placed on the content. Where the content includes any translated material, BMJ does not warrant the accuracy and reliability of the translations (including but not limited to local regulations, clinical guidelines, terminology, drug names and drug dosages), and is not responsible for any error and/or omissions arising from translation and adaptation or otherwise.

Open access This is an open access article distributed in accordance with the Creative Commons Attribution Non Commercial (CC BY-NC 4.0) license, which permits others to distribute, remix, adapt, build upon this work non-commercially, and license their derivative works on different terms, provided the original work is properly cited, appropriate credit is given, any changes made indicated, and the use is non-commercial. See: http://creativecommons.org/licenses/by-nc/4.0/.

\section{ORCID iDs}

Arul Earnest http://orcid.org/0000-0003-2693-5034

Kate Curtis http://orcid.org/0000-0002-3746-0348

\section{REFERENCES}

1 Gruen RL, Gabbe BJ, Stelfox HT, et al. Indicators of the quality of trauma care and the performance of trauma systems. Br J Surg 2012;99 Suppl 1:97-104.

2 Gabbe BJ, Simpson PM, Sutherland AM, et al. Improved functional outcomes for major trauma patients in a regionalized, inclusive trauma system. Ann Surg 2012;255:1009-15

3 Gabbe BJ, Lyons RA, Fitzgerald MC, et al. Reduced population burden of road transport-related major trauma after introduction of an inclusive trauma system. Ann Surg 2015;261:565-72.

4 West JG, Cales RH, Gazzaniga AB. Impact of regionalization. The orange County experience. Arch Surg 1983;118:740-4.

5 Lansink KWW, Leenen LPH. Do designated trauma systems improve outcome? Curr Opin Crit Care 2007;13:686-90.

6 Cameron PA, Gabbe BJ, Cooper DJ, et al. A statewide system of trauma care in Victoria: effect on patient survival. Med J Aust 2008;189:546-50.

7 Gabbe BJ, Biostat GD, Lecky FE, et al. The effect of an organized trauma system on mortality in major trauma involving serious head injury: a comparison of the United Kingdom and Victoria, Australia. Ann Surg 2011;253:138-43.

8 Lefering R, Huber-Wagner S, Nienaber U, et al. Update of the trauma risk adjustment model of the TraumaRegister DGUTM: the revised injury severity classification, version II. Crit Care 2014;18.
9 Bouamra O, Lesko MM. Outcome prediction modelling for trauma patients: a German perspective. Crit Care 2014;18:616.

10 Baker SP, O'Neill B, Haddon W, et al. The injury severity score: a method for describing patients with multiple injuries and evaluating emergency care. J Trauma 1974;14:187-96.

11 Committee on medical aspects of automotive safety. Rating the severity of tissue damage. I. The abbreviated scale. JAMA 1971;215:277-80.

12 Gennarelli TA, Wodzin E, eds. Abbreviated Injury Scale 2005 - Update 2008. Barrington, IL: AAAM, 2008.

13 Boyd CR, Tolson MA, Copes WS. Evaluating trauma care: the TRISS method. trauma score and the injury severity score. J Trauma 1987;27:370-8.

14 Champion HR, Copes WS, Sacco WJ, et al. The major trauma outcome study: establishing national norms for trauma care. $J$ Trauma 1990;30:1356-65.

15 Shiraishi A, Otomo Y, Yoshikawa S, et al. Derivation and validation of an easy-to-compute trauma score that improves prognostication of mortality or the trauma rating index in age, Glasgow coma scale, respiratory rate and systolic blood pressure (TRIAGES) score. Crit Care 2019;23:365

16 Wong TH, Krishnaswamy G, Nadkarni NV, et al. Combining the new injury severity score with an anatomical polytrauma injury variable predicts mortality better than the new injury severity score and the injury severity score: a retrospective cohort study. Scand J Trauma Resusc Emerg Med 2016;24:25.

17 Lefering R. Development and validation of the revised injury severity classification score for severely injured patients. Eur J Trauma Emerg Surg 2009;35:437-47.

18 Tohira H, Jacobs I, Mountain D, et al. Systematic review of predictive performance of injury severity scoring tools. Scand J Trauma Resusc Emerg Med 2012;20:63

19 Alfred Health. Australian trauma quality improvement (AusTQIP) collaboration (2019). Australia New Zealand trauma registry, management of the severely injured, 1 July 2017 to 30 June 2018. Melbourne, Victoria, 2019

20 Palmer CS, Gabbe BJ, Cameron PA. Defining major trauma using the 2008 abbreviated injury scale. Injury 2016;47:109-15.

21 Thomas A, Gennarelli E. The abbreviated injury scale 2005. update 2008. IL,USA, 2008.

22 Department of Health and Human Services Victoria. Victorian emergency minimum dataset (VEMD) manual 23rd edition 2018-19. version: 1.0, 2018. Available: https://www2healthvicgovau/hospitalsand-health-services/data-reporting/health-data-standards-systems/ data-collections/vemd [Accessed 13 Nov 2020].

23 Firth D. Bias reduction of maximum likelihood estimates. Biometrika 1993;80:27-38.

24 Harrell FE. Regression modeling strategies: with applications to linear models, logistic regression, and survival analysis. New York: Springer, 2001.

25 Fleiss J. Statistical methods for rates and proportions. 2 ed. New York: John Wiley, 1981.

26 Jurkovich GJ. Regionalized health care and the trauma system model. J Am Coll Surg 2012;215:1-11.

27 Newgard CD, Fildes JJ, Wu L, et al. Methodology and analytic rationale for the American College of surgeons trauma quality improvement program. J Am Coll Surg 2013;216:147-57.

28 Palmer CS, Davey TM, Mok MT, et al. Standardising trauma monitoring: the development of a minimum dataset for trauma registries in Australia and New Zealand. Injury 2013;44:834-41.

29 Verardi V, Croux C. Robust regression in Stata. Stata 2009;9:439-53.

30 Moore L, Hanley JA, Turgeon AF, et al. A new method for evaluating trauma centre outcome performance: TRAM-adjusted mortality estimates. Ann Surg 2010;251:952-8.

31 Cameron P. 30 years after the Major Trauma Outcome Study (MTOS) - Benchmarking trauma Centre outcomes, the Challenge continues. Injury 2020;51:588-9. 